\title{
Framework L para Desenvolvedores de mHealth no Contexto de Self-Care e Gamificação
}

\author{
Luma W. Oliveira ${ }^{1}$, Sérgio T. Carvalho ${ }^{1}$ \\ ${ }^{1}$ Instituto de Informática - Universidade Federal de Goiás (UFG) \\ Caixa Postal 131 - 74.001-970 - Goiânia - GO - Brazil \\ \{lumawanderley, sergio\}@inf.ufg.br
}

\begin{abstract}
This work investigates how gamification is used in health applications that aim to stimulate self-care. There is evidence in the literature that indicates the need to know the profile of the user to correctly use the elements of games, without extrapolating the main objective of the application, which is to treat health. To overcome this problem, a conceptual framework (method) was developed, consisting of two dimensions, Self-Care and Gamification, which incorporate some concepts and practices so that an application developer can design his application. At the evaluation stage, the mixed method was used, with questionnaire application and online interview with specialists. The results indicate that the framework helps developers marshal the mHealth application, primarily by encouraging engagement.
\end{abstract}

Resumo. Este trabalho investiga como a gamificação é utilizada nas aplicações de saúde que possuem como propósito estimular o self-care. Há evidências na literatura que indicam a necessidade de se conhecer o perfil do usuário para empregar corretamente os elementos de jogos, sem extrapolar o objetivo principal da aplicação, que é tratar da saúde. Para superar esse problema, foi desenvolvido um framework conceitual (método), composto por duas dimensões, Self-Care e Gamificação, que incorporam alguns conceitos e práticas para que um desenvolvedor de aplicações possa projetar sua aplicação. Na etapa de avaliação, foi utilizado o método misto, com aplicação de questionário e entrevista online com especialistas. Os resultados indicam que o framework auxilia desenvolvedores a gamificar a aplicação mHealth, principalmente por estimular o engajamento.

\section{Introdução}

O termo gamificação (do inglês, gamification) designa "o processo de tornar as atividades mais semelhantes a jogos" [Werbach 2014] para que o usuário se mantenha motivado em realizar atividades, sejam elas desgastantes, enjoativas, repetitivas ou mesmo dolorosas. A utilização da gamificação se torna propícia para o controle de doenças crônicas, devido às características dessas doenças, ou seja, de duração longa ou incerta e cujo tratamento envolva mudanças de estilo de vida, em um processo de cuidado contínuo.

Ao lidar com doença crônica, é necessária uma mudança no relacionamento entre paciente, equipe médica e familiares. Self-care, nesse termo, vem como uma complementação ao cuidado, onde o paciente cuida de sua própria saúde; e isso, impacta na adesão ao tratamento desse paciente [Camargo-Borges et al. 2008]. Nesse contexto, adesão significa uma escala de comprometimento do paciente ao tratamento médico 
[de Gusmão and Mion Jr 2006], sendo desejável que o paciente siga completamente o seu tratamento para que se mantenha saudável.

Neste trabalho, a gamificação é o meio para se alcançar engajamento do usuário, ou seja, comprometimento, envolvimento e interação com certa atividade. A gamificação apresenta, nesse sentido, recursos para o usuário se manter engajado na utilização da aplicação. Como a aplicação possui o objetivo de estimular self-care, o usuário deve realizar atividades de autocuidado para continuar interagindo com a aplicação. Portanto, a gamificação cria um vínculo entre o engajamento de utilização da aplicação e o engajamento que a aplicação quer estimular.

Em uma aplicação mHealth (mobile Health) direcionada para self-care, o paciente também pode ser visto como um jogador, aquele indivíduo que interage com o sistema e que se permite ser engajado, com o propósito de cuidar de sua saúde. Cada jogador se sente motivado de maneiras diferentes e isto reflete em seu comportamento e interações no sistema [Klock et al. 2016].

Apesar de a gamificação mostrar efeitos positivos para o engajamento e mudança de comportamento, estudos [Johnson et al. 2016] indicam que conforme é aplicada, pode não melhorar a usabilidade da aplicação, ou ainda, desestimular alguns usuários a utilizála. Um problema recorrente em seu uso é a tentativa dos desenvolvedores utilizarem os mesmos elementos de jogos para públicos diferentes e aplicações com objetivos diferentes [McCallum 2012]. Dessa forma, não há garantia que a gamificação esteja incentivando, verdadeiramente, o usuário, o que pode, portanto, comprometer a adesão ao tratamento [Miller et al. 2016].

Outro problema é o excesso de gamificação na aplicação de saúde, desfocando a aplicação de seu objetivo principal [Nunes et al. 2015]. Estes estudos indicam que a gamificação tem potencial para o engajamento do usuário, entretanto, devem haver diretrizes para que possa ser bem aproveitada na aplicação. Como aplicar a gamificação não é nada trivial e depende de diversos fatores, inclusive a subjetividade, não há um algoritmo que indique como utilizar esse recurso. Embora seja difícil avaliar se a gamificação é ou não efetiva, algumas características podem ser traçadas a fim de reduzir problemas que afetam a experiência do usuário, como por exemplo, evitar o uso excessivo de um elemento de jogo em detrimento a outros.

Nesse sentido, esse trabalho apresenta o Framework L, um método desenvolvido com o objetivo de orientar desenvolvedores de mHealth a gamificá-la. Ele indica os primeiros passos a serem analisados no projeto, a organização do conhecimento da área de self-care e, principalmente, defende dois pilares, o conhecimento do perfil do usuário (suas motivações) e a escolha adequada dos elementos de jogos.

\section{Framework L}

Para ampliar a visão do projeto e facilitar a construção de uma aplicação mHealth gamificada, foi desenvolvido o Framework L. A Figura 1 apresenta o diagrama de atividade que orienta o processo. O processo tem início com a definição de aspectos inicias do software, como o objetivo principal da aplicação, funções principais, dentre outras informações. 


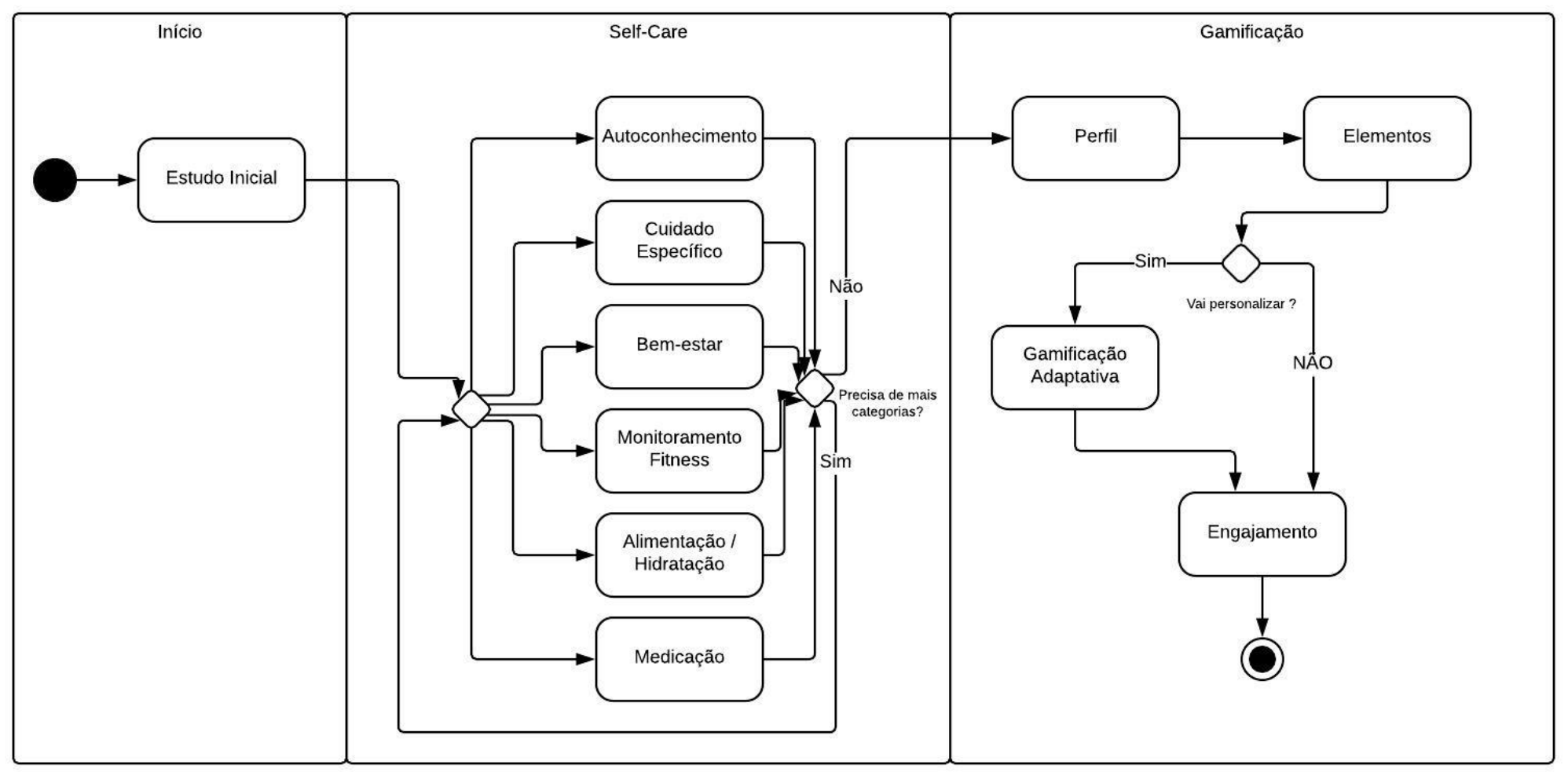

Figura 1. Diagrama de Atividade do Framework $L$

Em seguida, inicia-se o processo de escolha das categorias [Orem et al. 1995] da dimensão Self-Care, onde há a preocupação de conhecer o usuário em termos de sua saúde, considerando suas atividades e limitações. São elas: Autoconhecimento, Cuidados Específicos, Monitoramento Fitness, Alimentação / Hidratação, Medicação e Bem-estar. Cabe ao desenvolvedor, a partir do objetivo do software, escolher quais categorias ele vai trabalhar e o que deve ser considerado dentro de cada uma. É também aqui que se estabelecem as funções da aplicação e quais dados devem ser coletados, apresentados e manipulados.

Nesse ponto, o desenvolvedor inicia o projeto de gamificação já com um conhecimento mais amadurecido sobre o público-alvo e o objetivo da aplicação. A dimensão Gamificação possui o objetivo de auxiliar o desenvolvedor a conhecer o perfil de "jogador" do usuário, para uma escolha ciente dos elementos de jogos e a manipulação desses elementos na interface.

As classificações de perfil de jogadores podem auxiliar neste processo. Muitos destes classificadores disponibilizam os testes online, que retornam o tipo de jogador e sua motivação. O Framework L sugere a utilização da classificação de Marczewski [Marczewski 2015] por apresentar as principais características motivacionais e os elementos de jogos relevantes para cada tipo de jogador.

Existem vários elementos de jogos e diversas formas de relacioná-los. Seguindo a classificação de Marczewski, existem mais de 50 elementos que podem ser utilizados em gamificação, sendo eles para propósito geral (tema, feedback), progra- 
mados (por tempo, por evento), para atividades sociais (redes sociais, ranking), para exploração(escolhas, personalização), para desafios(aprendizagem, progressão), para altruísmo(compartilhamento de conhecimento, presentes), para inovação e mudança (anonimato, plataforma inovadora) e para recompensas (pontos, medalhas).

\section{Experimentos e Resultados}

O Framework L foi validado por um cenário onde se investigou sua aplicação em um contexto em que desenvolvedores criariam um mHealth para o controle de diabetes de estudantes de uma escola pública. O projeto para o desenvolvimento desse mHealth foi realizado passando por cada etapa do framework, indicando quais as principais decisões sobre os assuntos relacionados a self-care e a gamificação. Algumas dessas indicações estão listadas a seguir:

- Definir o objetivo principal do software

- Definir o público do software

- Definir o nível de autonomia do software

- Prever riscos ao usuário na utilização do software, se atentando para o apoio de um profissional da área médica

- Definir quais e como os dados serão apresentados, considerando as categorias da dimensão Self-care

- Definir os aspectos de jogo, seguindo as indicações da dimensão Gamificação

- Definir classificação de perfil de jogador

- Definir perfil de jogador do público-alvo

- Definir os elementos de jogos que serão utilizados

- Definir como as informações da dimensão Self-care contribuirão para os elementos de jogos

- Definir as estratégias de engajamento a médio e longo prazo

Após a descrição do cenário, foi abordado o método misto para avaliar o Framework L, utilizando-se de questionário e entrevista para a coleta de dados. Três especialistas das áreas de Interação Humano-Computador, Jogos e Informática em Saúde responderam um questionário online e participaram de uma entrevista online para um debate.

O questionário foi composto por treze afirmações sobre gamificação, self-care, engajamento e desenvolvimento de software, em que o avaliador deveria atribuir uma nota entre 1 e 5 (apontando seu nível de concordância). Duas questões abertas se referiram à relevância de ter um framework que apoia desenvolvedores e à relevância de se utilizar uma gamificação adaptativa.

O objetivo da entrevista foi avaliar aspectos do Framework L, como as descrições das dimensões. Os especialistas em Informática em Saúde avaliaram a dimensão SelfCare, principalmente, a descrição das categorias e a relação da área de saúde com gamificação. Os especialistas em Jogos ou Interação Humano Computador, diferentemente, avaliaram a dimensão Gamificação e os aspectos de desenvolvimento.

Os resultados apresentados levam em consideração as respostas obtidas no questionário e nas entrevistas, sendo predominantemente qualitativas onde os avaliadores fizeram observações como "a experiência divertida pode engajar um usuário a praticar selfcare", "as seis categorias da dimensão Self-Care são consistentes", "dimensão Self-Care 
poderia agregar outras categorias", "as opções do desenvolvedor não devem ser limitadas", "a capacidade de adaptar tem um grande potencial e pode ser reutilizada em várias situações diferentes", "poderia ressaltar outros aspectos que envolvem o desenvolvimento da aplicação".

Durante o trabalho, foi realizada uma revisão sistemática da literatura para averiguar o estado da arte da convergência dos temas de gamificação e self-care [Oliveira and Carvalho 2017]. Foi constatado que os elementos de jogos mais utilizados nos trabalhos foram pontuação, interação social e desafios, que são elementos mais voltados para públicos com motivação extrínseca.

Em relação a tecnologias em conjunto com gamificação e self-care, pode-se observar que a plataforma mobile foi a mais utilizada para o desenvolvimento das soluções, se destacando o uso de computação pervasiva e ubíqua. Dentre as categorias de problemas a serem cuidados, destacaram-se as doenças crônicas. Em relação ao perfil dos pacientes, a maioria dos trabalhos não aprofundaram no conhecimento do perfil do usuário.

Foram realizados ainda dois experimentos da etapa de Gamificação Adaptativa do Framework L. O principal objetivo desta etapa é o de personalizar e automatizar a aplicação mHealth para promover as motivações específicas de cada usuário. A adaptabilidade mencionada neste trabalho trata das modificações em links e conteúdos (como os elementos de jogos) com o objetivo de atender um modelo de usuário (perfil do usuário).

O primeiro experimento consistiu na utilização de uma rede neural artificial para que conforme o padrão de usabilidade do usuário no mHealth, o usuário pudesse ser classificado em um perfil de jogador (baseado na classificação de Marczewski). Diante do experimento, concluímos que os elementos de gamificação variam de usuário para usuário e podem se alterar conforme a mudança de usabilidade na aplicação.

O segundo experimento consistiu na aplicação de um teste de classificação de perfil de jogador, de maneira mais lúdica e coerente com uma aplicação gamificada. Foi possível aplicar e verificar os conceitos de gamificação tanto para aumentar o engajamento do usuário quanto para proporcionar uma experiência imersiva ao se realizar um teste de classificação de perfil de jogador. Essa pesquisa mostrou também que dependendo de como a gamificação é aplicada em um teste, pode interferir na autopercepção do usuário e influenciar em certas escolhas.

\section{Considerações Finais}

O Framework L incorpora várias características que são relevantes ao se considerar o desenvolvimento de um mHealth com a utilização de elementos de jogos. Dentre elas, a necessidade de conhecer o usuário, de valorizar a atividade a ser incentivada, não usar um elemento de jogo em detrimento de outros, etc.

Além disso, o framework disponibiliza uma etapa chamada de Gamificação Adaptativa que se mostra promissora em automatizar e personalizar a gamificação, embora ainda deva ser investigada em termos dos requisitos de usabilidade que podem interferir na descoberta do perfil de jogador.

Espera-se que o amadurecimento deste projeto simplifique para o desenvolvedor o processo de como utilizar gamificação para self-care e ao mesmo tempo, contribua para sua efetividade. 
Este trabalho gerou um artigo de revisão sistemática da literatura, publicado nos Anais do XVI Simpósio Brasileiro de Jogos e Entretenimento Digital [Oliveira and Carvalho 2017] e um resumo apresentado no II Workshop de Jogos e Saúde. Em 2018, um artigo que relata o processo de gamificação do teste de classificação de Marczewski [Oliveira and Carvalho 2018], foi publicado nos Anais do XVII Simpósio Brasileiro de Jogos.

\section{Referências}

Camargo-Borges, C., Japur, M., et al. (2008). Sobre a (não) adesão ao tratamento: ampliando sentidos do autocuidado. Texto Contexto Enferm, 17(1):64-71.

de Gusmão, J. L. and Mion Jr, D. (2006). Adesão ao tratamento-conceitos. Rev Bras Hipertens vol, 13(1):23-25.

Johnson, D., Deterding, S., Kuhn, K.-A., Staneva, A., Stoyanov, S., and Hides, L. (2016). Gamification for health and wellbeing: A systematic review of the literature. Internet Interventions, 6:89-106.

Klock, A. C. T., Flores, T. H., Gasparini, I., and da Silva Hounsell, M. (2016). Classificação de jogadores: Um mapeamento sistemático da literatura. Anais do Simpósio Brasileiro de Jogos e Entretenimento Digital. Porto Alegre: SBC.

Marczewski, A. (2015). Even ninja monkeys like to play: Gamification, game thinking \& motivational design. Gamified UK.

McCallum, S. (2012). Gamification and serious games for personalized health. Studies in health technology and informatics, 177(2012):85-96.

Miller, A. S., Cafazzo, J. A., and Seto, E. (2016). A game plan: Gamification design principles in mHealth applications for chronic disease management. Health Informatics $J$, 22(2):184-193.

Nunes, F., Verdezoto, N., Fitzpatrick, G., Kyng, M., Grönvall, E., and Storni, C. (2015). Self-care technologies in hci: Trends, tensions, and opportunities. ACM Transactions on Computer-Human Interaction (TOCHI), 22(6):33.

Oliveira, L. and Carvalho, S. T. (2017). Gamificação como estratégia para promover selfcare e engajar pacientes: Uma revisão sistemática da literatura. In SBGames 2017 Trilha Cultura.

Oliveira, L. and Carvalho, S. T. (2018). Gamificação do teste da classificação de marczewski. In SBGames 2018 - Trilha Cultura.

Orem, D. E., Taylor, S. G., and Renpenning, K. M. (1995). Nursing: Concepts of practice.

Werbach, K. (2014). (re) defining gamification: A process approach. In International conference on persuasive technology, pages 266-272. Springer. 